VOLUME 23 (2018) 143-152

DOI: $10.24330 /$ ieja.373656

\title{
FINITE-DIRECT-INJECTIVE MODULES
}

\author{
S. K. Maurya and A. J. Gupta \\ Received: 01 April 2017; Revised: 19 September 2017; Accepted: 04 October 2017 \\ Communicated by Abdullah Harmancı
}

\begin{abstract}
In this paper, we generalize the concept of direct-injective modules to finite-direct-injective modules. Various basic properties of these modules are studied. We show that the class of finite-direct-injective modules lies between the class of direct-injective modules and the class of simple-direct-injective modules. Also, we characterize semisimple artinian rings, $V$-rings and regular rings in terms of finite-direct-injective modules.
\end{abstract}

Mathematics Subject Classification (2010): 16D50, 16E50

Keywords: C2-module, C3-module, finite-direct-injective module, regular ring

\section{Introduction}

Throughout this paper, all rings are associative rings with unity and all modules are unitary right modules. For a right $R$-module $M, S=\operatorname{End}_{R}(M)$ denotes the endomorphism ring of $M$. For $\phi \in S, \operatorname{Ker}(\phi)$ and $\operatorname{Im}(\phi)$ stand for kernel and image of $\phi$, respectively. The notations $N \leq M, N \leq^{\text {ess }} M$ and $N \leq \oplus M$ means that $\mathrm{N}$ is a submodule, an essential submodule and a direct summand of $M$, respectively. $E(M)$ denotes the injective hull of $M$.

Y. Utumi [15] in a series of his papers on regular self injective rings observed three conditions on a ring which is satisfied if the ring is self injective. These conditions are currently known in the literature by $C 1, C 2$ and $C 3$ conditions and subsequently extended to modules as follows:

$(C 1)$ : every submodule of $M$ is essential in a direct summand of $M$.

$(C 2)$ : every submodule of $M$ which is isomorphic to a direct summand of $M$ is itself a direct summand of $M$.

$(C 3)$ : sum of any two direct summands of $M$ with zero intersection is again a direct summand of $M$.

The first author is thankful to IIT (Banaras Hindu University) Varanasi, India for providing the research facilities. 
The modules which satisfy the conditions $C 1, C 2$ and $C 3$ is known as $C 1$-module, $C 2$-module and $C 3$-module, respectively. These modules were studied by Mohamed and Müller in [9]. The concept of direct-injective modules which is the generalization of quasi-injective modules was introduced by W. K. Nicholson [10]. Nicholson et. al. [11] show that direct injective modules are equivalent to $C 2$-modules. Recently Camillo et. al. [2] generalize the concept of direct-injective modules to simple-direct-injective modules. A module $M$ is called simple-direct-injective if every simple submodule isomorphic to a direct summand of $M$ is itself a direct summand of $M$. In this paper, we introduce the concept of finite-direct-injective modules which is another generalization of direct-injective modules and it is interesting to note that these classes of modules lies between the class of direct-injective modules and the class of simple-direct-injective modules.

A module $M$ is called finite-direct-injective if every finitely generated submodule of $M$ isomorphic to a direct summand of $M$ is itself a direct summand of $M$. It is the generalization of direct-injective modules. We give an example of a finite-directinjective module that is not a direct-injective module. In Section 2 of this paper various basic properties of finite-direct-injective modules are studied. The class of finite-direct-injective modules is not closed under direct sum, even though direct summands of finite-direct-injective modules are finite-direct-injective. In Example 2.2 we will see that a direct sum of two finite-direct-injective modules need not be finite-direct-injective. Also, we give a sufficient condition for a finite-direct-injective module to be direct-injective. We also find a condition under which $C 3$-modules are finite-direct-injective.

In Section 3 of this paper, we characterize some well-known rings with the help of finite-direct-injective modules. B. L. Osofsky [12] proved that a ring $R$ with property that all its cyclic right modules are injective is semisimple artinian. Here we give a characterization of semisimple artinian ring in terms of finite-directinjective modules. A ring $R$ is called a right $V$-ring if every simple right $R$-module is injective. It is shown that a ring is right $V$-ring if and only if every finitely cogenerated $R$-module is finite-direct-injective. According to G. Lee et. al. [7], a right $R$-module $M$ is called dual Rickart if, $\forall \phi \in S, \phi(M)=\operatorname{Im}(\phi)=e M$ for some $e^{2}=e \in S$. A module $M$ is said to have the summand sum property $(S S P)$, if the sum of any two direct summands of $M$ is a direct summand of $M$ (see for details, [1], [5]). For a semihereditary ring $R$, it is shown that every finitely generated projective $R$-module is finite-direct-injective if and only if every finitely generated projective $R$-module is dual Rickart if and only if every finitely generated projective 
$R$-module satisfies summand sum property if and only if $R$ is a regular ring. We also characterize rings $R$ for which every singular right $R$-module is finite-directinjective.

\section{Finite-direct-injective modules}

Here we introduce the concept of finite-direct-injective modules as a generalization of direct-injective modules with counter example and discuss some properties of finite-direct-injective modules.

Definition 2.1. A module $M$ is called finite-direct-injective if every finitely generated submodule of $M$ isomorphic to a direct summand of $M$ is itself a direct summand of $M$. A ring $R$ is called right finite-direct-injective if the right $R$-module $R$ is finite-direct-injective.

Example 2.2. (1) Every direct-injective module is finite-direct-injective but the converse need not be true. Here we give an example of a finite-direct-injective module that is not direct-injective. Let $R$ be a von Neumann regular ring which is not semisimple. For instance, the endomorphism ring of an infinite dimensional vector space. As $R$ is not semisimple, ${ }_{R} R$ has infinite Goldie dimension. So it contains an infinite direct sum $N=\bigoplus_{n \in N} R r_{n}$ of non zero left ideal. Note that $N$ is not a direct summand of $R$ as it is not finitely generated. Let ${ }_{R} M=R^{\mathbb{N}}$, a countable direct sum of copies of the ring $R$ and $N$ be the left ideal of $R$ included in the first copy of the ring inside $M=R^{\mathbb{N}}$. Clearly any finitely generated submodule of $M$ is a direct summand since $R$ is regular. Hence $M$ is finite-direct-injective but it is not direct-injective because if we define an R-homomorphism $f: N \rightarrow M$ by $f\left(\sum_{i \in N} x_{i} r_{i}\right)=\left(x_{1} r_{1}, x_{2} r_{2}, \ldots, x_{n} r_{n}, \ldots\right)$. Let $K=\operatorname{Im}(f)$, then $K$ is clearly a direct summand of $M$ isomorphic to $N$ but $N$ is not a direct summand of $M$.

(2) A module whose finitely generated submodule is a direct summand is trivially finite-direct injective. In particular every strongly regular [14] and every finitely generated projective modules over a von Neumann regular ring are finite-directinjective.

(3) Lee et. al. [8], defined a module $M$ to be automorphism invariant if $\alpha(M) \leq M$ for every automorphism $\alpha$ of the injective hull of $M$. In [4, Theorem 16], it was shown that a module $M$ is automorphism invariant if and only if it is pseudoinjective. By [3, Theorem 2.6], every pseudo-injective module as well as every automorphism invariant module is a C2 (direct-injective) module, and hence is a finite-direct-injective module. However every finite-direct-injective module may not be automorphism invariant. For example, if a ring $R$ is von Neumann regular such 
that $R$ is not a clean ring, then $R_{R}$ is direct-injective and hence finite-direct-injective but it is not automorphism invariant.

Proposition 2.3. Every direct summand of a finite-direct-injective module is a finite-direct-injective module.

Proof. Let $M$ be a finite-direct-injective module and $N$ be a direct summand of $M$. Let $X$ be a finitely generated submodule of $N$ which is isomorphic to a direct summand $P$ of $N$. We have to show that $X$ is also a direct summand of $N$. Since $P$ is a direct summand of $N$ and $N$ is a direct summand of $M$, we have $P$ is a direct summand of $M$. So $X \cong P \leq \oplus M$. Since $M$ is finite-direct-injective, $X$ is a direct summand of $M$. Let $M=X \bigoplus Y$ for some $Y \leq M$. By modular law $N=N \cap M=N \cap(X \bigoplus Y)=X \bigoplus(N \cap Y)$. Thus $X$ is a direct summand of $N$.

It is interesting to examine whether an algebraic property is inherited by direct sums. The examples given below shows that a direct sum of two finite-directinjective modules need not be finite-direct-injective.

Example 2.4. (1) Let $S$ be a simple $R$-module that is not injective, so it is easy to see that $S$ and its injective hull $E(S)$ are finite-direct-injective but $S \bigoplus E(S)$ is not finite-direct-injective.

(2) Let

$$
R=\left[\begin{array}{cc}
F & F \\
0 & F
\end{array}\right], \quad A=\left[\begin{array}{cc}
F & F \\
0 & 0
\end{array}\right], \quad B=\left[\begin{array}{ll}
0 & 0 \\
0 & F
\end{array}\right]
$$

where $F$ is a field. Then $R_{R}=A_{R} \oplus B_{R}$. Both $A_{R}$ and $B_{R}$ are finite-directinjective, but $R_{R}$ is not a finite-direct-injective module.

Now let us see how finite-direct-injective modules correlate with direct-injective modules and simple-direct-injective modules, as defined. We have the following hierarchy:

Proposition 2.5. The following implications hold and are irreversible:

Direct-injective $\Longrightarrow$ finite-direct-injective $\Longrightarrow$ simple-direct-injective.

Proof. This is clear from the definitions.

Remark 2.6. In general none of the implication given in above proposition is an equivalence. For example $\mathbb{Z}$ as a $\mathbb{Z}$-module is simple-direct-injective but it is not finite-direct-injective as well as direct-injective. 
The next proposition gives a sufficient condition for a finite-direct-injective module to be direct-injective.

Proposition 2.7. Let $M$ be a finitely generated right $R$-module. Then $M$ is finitedirect-injective if and only if $M$ is direct-injective. In particular, a ring $R$ is right finite-direct-injective if and only if it is right direct-injective.

Proof. Let $M$ be a finite-direct-injective module and $N$ be any submodule of $M$ such that $N \cong P \leq \oplus M$. Since $M$ is finitely generated, therefore $P$ is finitely generated and so $N$ is finitely generated and becomes direct summand of $M$ as $M$ is finite-direct-injective. The converse is clear from the definition. Since any ring $R$ is cyclic as an $R$-module, therefore $R$ is finite-direct-injective if and only if $R$ is direct-injective.

The next proposition is an important tool which is used to develop some properties of finite-direct-injective modules and also help in the characterization of various rings in terms of finite-direct-injective modules.

Proposition 2.8. Let $M=M_{1} \bigoplus M_{2}$ for some submodules $M_{1}$ and $M_{2}$ with $M_{1}$ finitely generated. If $M$ is a finite-direct-injective module and $f: M_{1} \rightarrow M_{2}$ is a homomorphism with $\operatorname{Ker}(f) \leq \oplus M_{1}$, then $\operatorname{Im}(f) \leq \oplus M_{2}$.

Proof. Let $f: M_{1} \rightarrow M_{2}$ be a module homomorphism with $\operatorname{Ker}(f) \leq \oplus M_{1}$, say $M_{1}=\operatorname{Ker}(f) \bigoplus N$. Then by fundamental theorem of module homomorphisms, $\operatorname{Im}(f) \cong M_{1} / \operatorname{Ker}(f) \cong N$. Since $M_{1}$ is finitely generated so, $M_{1} / \operatorname{Ker}(f)$ and hence $\operatorname{Im}(f)$ is finitely generated. Also given that $M$ is finite-direct-injective so, $\operatorname{Im}(f)$ is a direct summand of $M$ as $N$ is a direct summand. Since $\operatorname{Im}(f) \leq M_{2} \leq \oplus$ $M, \operatorname{Im}(f) \leq \oplus M_{2}$.

Corollary 2.9. Let $M$ be a finite-direct-injective module, $M=M_{1} \bigoplus M_{2}$ for some submodules $M_{1}$ and $M_{2}$ with $M_{1}$ finitely generated, and $f: M_{1} \rightarrow M_{2}$ be a monomorphism. Then $\operatorname{Im}(f) \leq \oplus M_{2}$.

Proof. The proof is clear from Proposition 2.8.

Corollary 2.10. Let $M$ be a finitely generated module and $M \oplus E(M)$ be finitedirect-injective. Then $M$ is injective.

Proof. Since the inclusion map $i: M \rightarrow E(M)$ is clearly a monomorphism then by Corollary 2.9, $i(M)=M \leq \oplus E(M)$. Thus $M=E(M)$ and hence $M$ is injective. 
Proposition 2.11. If every 2-generated right $R$-module is finite-direct-injective, then every finite dimensional right $R$-module is injective.

Proof. To show that every finite dimensional right $R$-module is injective we have to show that every uniform module is injective. Let $L$ be a uniform module and for any $0 \neq x \in E(L), 0 \neq P \leq x R$ and take $0 \neq y \in P$. Then $x R \oplus y R$ is a finite-direct-injective module. By Corollary 2.9, $y R \leq \oplus x R$. But as $x R$ is indecomposable $y R=x R$ and so $P=x R$. Thus every cyclic submodule of $x R$ is a direct summand hence $x R$ is semisimple and so $E(L)$ is semisimple. Thus $L=E(L)$ is injective as desired.

Proposition 2.12. Let $M$ be a finite dimensional, direct-injective module. Then $\operatorname{End}_{R}(M)$ is semilocal.

Proof. Since $M$ is finite dimensional to prove that $\operatorname{End}_{R}(M)$ is semilocal we need to show that every monomorphism $\alpha: M \rightarrow M$ is an isomorphism. Since $\alpha(M) \cong$ $M \leq \oplus M$ and $M$ is direct-injective, therefore $\alpha(M) \leq \oplus M$. But $\alpha(M) \leq{ }^{\text {ess }} M$ as $M$ is finite dimensional. Hence $\alpha(M)=M$, so $\alpha$ is an isomorphism, as desired.

Corollary 2.13. Let $M$ be a finitely generated, finite dimensional, finite-directinjective module. Then $\operatorname{End}_{R}(M)$ is semilocal.

Proof. The proof follows easily from Propositions 2.7 and 2.12 .

It is observed that $\mathbb{Z}$ as a $\mathbb{Z}$-module is a $C 3$-module but it is not finite-directinjective. Thus every $C 3$-module need not be finite-direct-injective. In the next proposition we find the condition under which $C 3$-modules are finite-direct-injective.

Proposition 2.14. The following statements hold:

(1) If $M$ is a finite-direct injective module, then for any two direct summands $A$ and $B$ of $M$ with $A \cap B=0$ and $B$ finitely generated, $A \oplus B \leq \oplus M$.

(2) If $M \bigoplus M$ is a C3-module, then $M$ is a finite-direct-injective module.

(3) Any direct sum of injective modules is finite-direct-injective.

Proof. (1) Suppose $M$ is a finite-direct-injective module and $A, B \leq \oplus M$ with $A \cap B=0$ and $B$ is finitely generated. Write $M=A \oplus K$ for some $K \leq M$ and let $\pi: M \rightarrow K$ be the natural projection. Since $B$ is a finitely generated direct summand of $M$ with $A \cap B=0, A \oplus B=A \oplus \pi(B)$ and $\pi(B) \cong B \leq \oplus M$. Since $M$ is a finite-direct-injective module, $\pi(B) \leq \oplus M$. Write $M=\pi(B) \oplus C$ for some $C \leq M$, then $K=K \cap M=K \cap(\pi(B) \oplus C)=\pi(B) \oplus(C \cap K)$. Thus $M=A \oplus K=A \oplus \pi(B) \oplus(C \cap K)=A \oplus B \oplus(C \cap K)$, as required. 
(2) Let $M \oplus M$ be a $C 3$-module and $K$ be a finitely generated submodule of $M$ such that $K \cong L \leq \oplus M$. We need to show that $K \leq \oplus M$. Write $M=L \oplus N$ for some $N \leq M$. Since $M \bigoplus M=(L \bigoplus N) \bigoplus M=L \bigoplus(M \bigoplus N)$ is a $C 3$-module, and if we take $\sigma: K \rightarrow L$ as the preceeding isomorphism, $\sigma^{-1}: L \rightarrow M \rightarrow M \bigoplus N$ splits by Lemma 3.2 of [2]. Hence $K=\sigma^{-1}(L) \leq \oplus M$.

(3) Let $M=\bigoplus_{i \in \iota} E_{i}$ be an arbitrary direct sum of injective modules $E_{i}$. Let $A \cong B \leq \oplus M$ where $A$ and $B$ are finitely generated submodules of $M$. Since $B$ is finitely generated, $B \leq \oplus\left(\bigoplus_{i \in F} E_{i}\right)$ for some finite subset $F \subset \iota$. Since finite direct sums of injective modules are injective, $B$ is injective and since $A \cong B, A$ is injective and so $A \leq \oplus M$, as required.

Two modules $A$ and $B$ are called subisomorphic if $A$ isomorphic to a submodule of $B$ and $B$ is isomorphic to a submodule of $A$. According to Goldie [6], two modules are subisomorphic if each has a monomorphism into the other one. A module $M$ is called directly finite if it is not isomorphic to a proper direct summand of itself.

Proposition 2.15. Let $M$ be a finitely generated $R$-module such that $M=A \oplus B$ is a finite-direct-injective module, where $A$ and $B$ are subisomorphic. If either $A$ or $B$ is directly finite, then $A \cong B$.

Proof. Since $M$ is a finitely generated $R$-module and $M=A \bigoplus B, A$ and $B$ are also finitely generated. Let $\alpha: A \rightarrow B$ and $\beta: B \rightarrow A$ be monomorphisms. Since $\beta(B) \cong B \leq \oplus M$ and $M$ is a finite-direct-injective module, $\beta(B) \leq \oplus M$, but $\beta(B) \leq A$, so $\beta(B) \leq \oplus A$. Let $A=\beta(B) \oplus T$ for a submodule $T \leq A$. Now as $\alpha \beta: B \rightarrow B$ is a monomorphism, so $\alpha \beta(B) \cong B \leq \oplus M$ and $M$ is a finite-directinjective module, therefore $\alpha \beta(B) \leq \oplus B$. Let $B=\alpha \beta(B) \oplus L$ for a submodule $L$ of $B$. According to our assumption let $B$ be directly finite and since $B \cong \alpha \beta(B)$, $L=0$. Thus $B=\alpha \beta(B)=\alpha(A)$, so $\alpha$ is an isomorphism between $A$ and $B$ as required.

\section{Characterization of rings using finite-direct-injective modules}

In this section, we characterize some well-known rings with the help of finitedirect-injective modules in which Corollary 2.10 play an important role. In the next result, we characterize semisimple artinian rings in terms of finite-direct-injective modules.

Proposition 3.1. The following conditions are equivalent for a ring $R$ :

(1) $R$ is semisimple artinian.

(2) Every $R$-module is finite-direct-injective. 
Proof. (1) $\Longrightarrow(2)$ This is clear.

$(2) \Longrightarrow(1)$ Let $N$ be a cyclic $R$-module. By (2), $N \bigoplus E(N)$ is a finite direct injective module. Hence by Corollary $2.10, N$ is injective. Thus according to Osofsky Theorem [12], $R$ is semisimple artinian.

Now we characterize $V$-rings in terms of finite-direct-injective modules.

Theorem 3.2. The following conditions are equivalent for a ring $R$ :

(1) $R$ is right $V$-ring.

(2) Every finitely cogenerated $R$-module is finite-direct-injective.

Proof. (1) $\Longrightarrow(2)$ Let $R$ be a right $V$-ring. Then every finitely cogenerated module is injective by Theorem 23.1 of [16]. Hence every finitely cogenerated $R$ module is finite-direct-injective.

$(2) \Longrightarrow(1)$ To show that $R$ is a right $V$-ring we have to show that every simple $R$-module is injective. Let $M$ be a simple $R$-module. Then $M \bigoplus E(M)$ is finitely cogenerated. By (2), it is a finite-direct-injective module. Hence by Corollary 2.10, $M$ is injective and thus $R$ is a right $V$-ring.

The next theorem characterizes regular rings in terms of finite-direct-injectivity.

Theorem 3.3. The following conditions are equivalent for a semihereditary ring $R$ :

(1) Every finitely generated projective $R$-module is finite-direct-injective.

(2) Every finitely generated projective R-module is dual Rickart.

(3) Every finitely generated projective $R$-module has SSP.

(4) Every finitely generated submodule of a finitely generated projective $R$ module is a direct summand.

(5) $R$ is a regular ring.

Proof. (1) $\Longrightarrow \quad(2)$ Let $M$ be a finitely generated projective $R$-module and $S=\operatorname{End}(M)$. To show that $M$ is dual Rickart we have to show that for any $s \in S, s(M) \leq \oplus M$. Since $M \oplus s(M)$ is finitely generated projective, by (1), it is finite-direct-injective. Hence by Corollary 2.10, $s(M) \leq \oplus M$, as desired.

(2) $\Longrightarrow$ (3) Every dual Rickart module satisfies SSP [7, Proposition 2.11]. Therefore by (2), every finitely generated projective $R$-module has SSP.

(3) $\Longrightarrow$ (4) Let $\mathrm{N}$ be a finitely generated submodule of a finitely generated projective $R$-module $M$. Then $N \bigoplus M$ is finitely generated projective and so by (3), it has SSP. Therefore $N \leq \oplus M$. 
(4) $\Longrightarrow$ (5) Since $R$ is a finitely generated projective $R$-module, by (4), every cyclic right ideal of $R$ is a direct summand of $R$. Hence $R$ is a regular ring.

$(5) \Longrightarrow$ (1) Let $R$ be a regular ring and $M$ a finitely generated projective $R$-module. Then every finitely generated submodule of $M$ is a direct summand, therefore $M$ is trivially finite-direct-injective.

Rings $R$ for which every singular right $R$-modules are injective are called right SI-rings. In the next proposition, we characterize right SI-ring with the help of finite-direct-injective modules.

Proposition 3.4. The following conditions are equivalent for a ring $R$ :

(1) $R$ is a right SI-ring.

(2) Every singular right $R$-module is finite-direct-injective.

Proof. (1) $\Longrightarrow(2)$ Since $R$ is a right SI-ring, every singular right $R$-module is injective, therefore every singular right $R$-module is finite-direct-injective.

$(2) \Longrightarrow(1)$ Let $M$ be a cyclic singular right $R$-module, then it is easy to see that $M \bigoplus E(M)$, where $E(M)$ is the injective hull of $M$, is singular and by hypothesis it is finite-direct-injective. So by Corollary 2.10, $M$ is injective. Thus every cyclic singular right $R$-module is injective. Hence by [13, Corollary 5], every singular right $R$-module is injective. Thus $R$ is a right SI-ring.

Acknowledgment. The authors would like to thank the referee for the valuable suggestions and comments.

\section{References}

[1] M. Alkan and A. Harmanci, On summand sum and summand intersection property of modules, Turkish J. Math., 26(2) (2002), 131-147.

[2] V. Camillo, Y. Ibrahim, M. Yousif and Y. Zhou, Simple-direct-injective modules, J. Algebra, 420 (2014), 39-53.

[3] H. Q. Dinh, A note on pseudo-injective modules, Comm. Algebra, 33(2) (2005), 361-369.

[4] N. Er, S. Singh and A. K. Srivastava, Rings and modules which are stable under automorphisms of their injective hulls, J. Algebra, 379 (2013), 223-229.

[5] J. L. Garcia, Properties of direct summands of modules, Comm. Algebra, 17(1) (1989), 73-92.

[6] A. W. Goldie, Torsion-free modules and rings, J. Algebra, 1 (1964), 268-287.

[7] G. Lee, S. T. Rizvi and C. S. Roman, Dual Rickart modules, Comm. Algebra, 39(11) (2011), 4036-4058. 
[8] T. K. Lee and Y. Zhou, Modules which are invariant under automorphisms of their injective hulls, J. Algebra Appl., 12(2) (2013), 1250159 (9 pp).

[9] S. H. Mohamed and B. J. Müller, Continuous and Discrete Modules, London Mathematical Society Lecture Note Series, 147, Cambridge University Press, Cambridge, 1990.

[10] W. K. Nicholson, Semiregular modules and rings, Canad. J. Math., 28(5) (1976), 1105-1120.

[11] W. K. Nicholson and Y. Zhou, Semiregular morphisms, Comm. Algebra, 34(1) (2006), 219-233.

[12] B. L. Osofsky, Rings all of whose finitely generated modules are injective, Pacific J. Math., 14 (1964), 645-650.

[13] B. L. Osofsky and P. F. Smith, Cyclic modules whose quotients have all complement submodules direct summands, J. Algebra, 139(2) (1991), 342-354.

[14] V. S. Ramamurthi and K. M. Rangaswamy, On finitely injective modules, J. Austral. Math. Soc., 16 (1973), 239-248.

[15] Y. Utumi, On continuous rings and self injective rings, Trans. Amer. Math. Soc., 118 (1965), 158-173.

[16] R. Wisbauer, Foundations of Module and Ring Theory, Algebra, Logic and Applications, 3, Gordon and Breach Science Publishers, Philadelphia, PA, 1991.

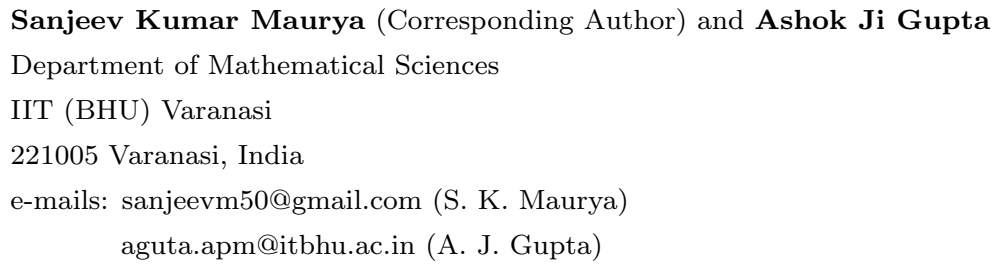

